\title{
UN ASPECTO OLVIDADO EN LAS CONTRATACIONES DIRECTAS POR CAUSAL DE EMERGENCIA, COVID-19: LA LOGÍSTICA
}

\section{UM ASPECTO ESQUECIDO NAS CONTRATACÕES DIREITOS EM RAZÃO DA PANDEMIA DA COVID-19: A LOGÍSTICA}

\section{A FORGOTTEN ASPECT IN DIRECT HIRING DUE TO THE COVID-19 PANDEMIC: THE LOGISTICS}

Raquel Alianak ${ }^{1}$

Recebido em: 28/10/2021 Aceito em: 28/10/2021 raquelalianak@hotmail.com

Abstracto: Este trabajo aborda la contratación directa por parte de la Administración Pública en tiempos de pandemia COVID-19 en Argentina. Inicialmente, se abordan estrategias innovadoras de gestión pública. Por ello, se analiza la contratación directa en tiempo de pandemia. Por último, se busca estudiar la gestión, planificación y logística de las actuaciones de las áreas de compras de la Administración Pública. Así, la pregunta es: ¿qué logistia se está implementando para la contratación directa en tiempos de pandemia? La importancia del estudio radica en la importancia de entender la actuación de la administración en tiempos de pandemia, ante la situación de emergencia.

Palavras-chave: Administración Pública. Pandemia. Contratación directa

Resumo: O presente trabalho aborda a contratação direta pela Administração Pública em tempos de pandemia da COVID-19 na Argentina. Inicialmente, aborda-se estratégias inovadoras de gestão pública. Por conseguinte, analisa-se as contratações diretas em tempo de pandemia. Por fim, buscase estudar a gestão, planejamento e logística das ações das áreas de compras da Administração Pública. Sendo assim, pergunta-se: qual a logístia está sendo implementada para contratação direta em tempos de pandemia? A importância do estudo reside na relevância de compreender a atuação da administração em tempos de pandemia, tendo em vista a situação emergencial.

Palavras-chave: Administração Pública. Pandemia. Contratação Direta.

Abstract: This paper addresses the direct contracting by the Public Administration in times of pandemic covid-19 in Argentina. Initially, innovative public management strategies are addressed. Therefore, direct hiring in pandemic time is analysed. Finally, we seek to study the management,planning and logistics of the actions of the procurement areas of the Public Administration. Thus, the question is: what is the logistia being implemented for direct contracting in times of pandemic? The importance of the study lies in the importance of understanding the action of the administration in times of pandemic, in view of the emergency situation.

Keywords: Public Administration. Pandemic. Direct Hiring.

\section{INTRODUCCIÓN}

En el presente trabajo se estudia la contratación directa por parte de la Administración Pública en tiempos de pandemia.

Nesste context, se pregunta: ¿cuál es la logistia que se está implementando en Argentina, en materia de gestión pública, para la contratación directa en tiempos de pandemia?

Para responder a esta pregunta, se abordan inicialmente prácticas innovadoras en materia de gestión pública y estrategias de contratación directa en Argentina.

\footnotetext{
${ }^{1}$ Universidad Nacional de Rosário - Rosário - Argentina
} 
En segundo lugar, analizamos la normativa sobre contratación directa en el periodo de emergencia de la pandemia del COVID-19 y el régimen especial de contratación de bienes y servicios en el periodo.

Finalmente, se recogen recomendaciones internacionales de transparencia que implican la contratación de bienes y servicios públicos en tiempos de pandemia, que tienen como objetivo mejorar el régimen de contratación.

La importancia del estudio radica en la necesidad de comprender y señalar las posibles brechas en el sistema de gestión pública en tiempos de pandemia, con respecto a la logística de contratación directa de bienes y servicios públicos.

\section{ESTRATEGIAS INNOVADORAS EN GESTIÓN PÚBLICA}

La reciente aprobación de la Carta Iberoamericana de Innovación en la Gestión Pública, por la "XIX Conferencia Iberoamericana de Ministras y Ministros de la Administración Pública y Reforma del Estado", llevada a cabo en Andorra, el 08 de octubre de 2020, nos conduce inexorablemente a repensar las modalidades de actuación de las Administraciones Públicas, en aspectos multifacéticos, que se proyectan ya sea en el procedimiento administrativo, y/o en el proceso administrativo, y/o en la contratación pública, y/o en la participación ciudadana y sus nuevos mecanismos, y/o en un nuevo modo de gestión de las políticas públicas, entre otros tantas temáticas necesariamente involucradas.

Esta óptica está orientada a una gestión pública con mirada hacia la cultura de la innovación, con el fin de brindar soluciones inéditas y creativas a los problemas y necesidades no solo tradicionales sino nuevas de los ciudadanos y de la sociedad, mediante herramientas indispensables para hacerlo del modo más eficaz.

La innovación en el ámbito público enfoca nuevas modalidades de gestionar; nuevas estrategias que se deben implementar para lograr una sociedad más involucrada en la gobernanza colectiva de los asuntos públicos; y la inclusión de la tecnología, pues la Administración Pública innovadora va de la mano de la Administración Pública inteligente, que analiza e interpreta datos desde lo tecnológico y desde el conocimiento humano- en procura de promover mejor calidad de vida, un desarrollo sostenible dentro de los Objetivos de la Agenda 2030 de Naciones Unidas, así como eficaces y modernos sistemas de control y de evaluación de las políticas públicas y del desempeño de los servidores públicos, focalizando la gestión por resultados y -además- en base a nuevos criterios (creatividad, pensamiento crítico, orientación al ciudadano, resolución efectiva de problemas, cooperación y trabajo en equipo).

La nueva Carta Iberoamericana de Innovación del mes de Octubre del corriente año, nos habla del valor de la inteligencia colectiva, como el enriquecimiento mutuo de personas y grupos (tanto del sector público como del privado) con relación a un tema o a la resolución de un problema determinado, a fin de congregar todos esos micro saberes, para lograr soluciones eficaces; y por supuesto de la Inteligencia artificial y también de la robótica, en beneficio del interés general, a ser utilizadas teniendo en cuenta los valores y los principios de los derechos humanos y del bienestar y de la seguridad jurídica. 
Por ende, la innovación -entonces- en el modo de gestionar impone una VISIÓN ESTRATÉGICA por parte de la Administración Pública, lo cual implica observar la realidad actual, y al mismo tiempo tener una perspectiva de futuro, realizando un mapeo de riesgos. Esta visión estratégica involucra:

(i) un actuar ANTICIPATORIO, para anticipar el efecto de las acciones venideras, a través de una visión a largo plazo, orientada al logro de objetivos puntuales;

(ii) un actuar ADAPTATIVO, para poder reaccionar eficazmente ante hechos inesperados; y por último

(iii) un actuar PROACTIVO, elaborando potenciales escenarios de futuro.

Este faro es el que debe, así, incidir en las decisiones de política pública que se adopten, en los distintos niveles jurisdiccionales, realizando un ANÁLISIS DE PROSPECTIVA, ante los cambios relevantes y rápidos de carácter tecnológico, económico y social de este Siglo.

Precisamente, una de las DIMENSIONES de esa innovación en la gestión pública, a fin de hacer ANCLAJE EN ESTE ÚLTIMO EJE comentado, es la relacionada con la CONTRATACIÓN PÚBLICA.-

Pues es en el ámbito contractual -incidido como se encuentra el mundo entero por la emergencia sanitaria del COVID-19- donde debe ser prioritario, por parte de la Administración Pública, hoy más que nunca, una VISIÓN DE PROSPECTIVA, que implique un actuar ADAPTATIVO, para poder reaccionar de modo eficaz ante circunstancias imprevistas; $y$-además- a esta altura de los acontecimientos, un accionar ANTICIPATORIO, para prever -y en su caso- regular consecuencias y efectos necesarios a tener en cuenta derivados de contrataciones que debe inexorablemente realizar, para que los fines involucrados en aquellas puedan eficazmente lograrse.

Resulta evidente asimismo que se requiere un actuar de modo PROACTIVO y con la DEBIDA DILIGENCIA, teniendo en mente posibles escenarios de futuro.

En consecuencia, un tema que estimo necesario a tener en cuenta, y que ha sido olvidado por la Administración pública nacional en Argentina e incluso la provincial, en una gestión que no fue ni anticipatoria ni proactiva, es el de la LOGíSTICA, relacionada con los bienes y servicios adquiridos por contratación directa en el curso de la pandemia, extensible a las adquisiciones que continuarán realizándose como consecuencia de los efectos del COVID-19.

\section{CONTRATACIÓN DIRECTA POR PARTE DE LA ADMINISTRACIÓN PÚBLICA EN TIEMPOS DE PANDEMIA}

La pandemia en el mundo entero determinó que las Administraciones Públicas efectúen contrataciones directas, las cuales demostraron -en algunos casos- no contar con la eficiencia, transparencia, agilidad y confiabilidad que eran esperables, precisamente a la luz de las finalidades que las inspiraron y los resultados que debían lograrse, puntual y prontamente.

En muchos países, esas contrataciones se realizaron enmarcadas en el régimen jurídico vigente de contratación pública; en particular, conforme a los procedimientos y notas esenciales de la contratación directa por "causal de emergencia". 
Pero también encontramos ejemplos, en los que se dictaron normas especiales de contratación pública por la emergencia sanitaria de la pandemia del Coronavirus.

En la República Argentina, el régimen general de contratos públicos nacionales, regulado por el Decreto $N^{\circ} 1023 / 01$ y su Decreto reglamentario $N^{\circ} 1030 / 16$ contempla como uno de los supuestos excepcionales de contratación directa (Art. 25 apartado "d", inciso "5"), los casos de "emergencia o urgencia". El Decreto 1023/01 únicamente requiere: (i) que el supuesto sea objetivo y se encuentre debidamente acreditado en las actuaciones; (ii) que impida realizar otro procedimiento de selección en tiempo oportuno, y (iii) que sea aprobado por la máxima autoridad de cada ente.

A dicha escueta normativa, cabe solo agregar la aclaración formulada por la reglamentación en torno al alcance de la expresión "razones de urgencia", y al de "razones de emergencia", estas últimas como accidentes, fenómenos climáticos o sucesos creadores de situación de peligro o desastre que requiera acción inmediata, y que comprometan la vida, integridad física, salud, seguridad de la población o funciones esenciales del Estado.

Se expresa, finalmente, que esas situaciones deben ser imprevisibles, pues si no lo son, deberán establecerse las responsabilidades emergentes como consecuencia de no utilizar un procedimiento de contratación competitivo en tiempo oportuno.

Este breve marco normativo general de contratación directa por urgencia y/o emergencia evidencia amplias lagunas regulatorias; y resulta insuficiente para ser aplicado conforme los únicos estándares contemplados en la regulación

El régimen general descrito anteriormente no fue utilizado en el contexto de la pandemia, pues en Argentina fue dictado un RÉGIMEN ESPECIAL para contratar bienes y servicios imprescindibles para satisfacer necesidades de naturaleza hospitalaria y social que la situación actual requería y requiere.

Sin embargo, ni en el régimen general de contratación pública, ni en el especial dictado con motivo de la pandemia, se ha tratado el tópico de la LOGÍSTICA para las contrataciones directas por causal de emergencia.

A qué nos estamos refiriendo concretamente?

A los medios necesarias para organizar el servicio de distribución de los insumos y bienes en tiempo urgente y oportuno, acorde con las necesidades que motivaron la contratación.-

Es decir, a las actividades logísticas necesarias para cubrir la gestión y planificación de acciones de las áreas de adquisiciones de la Administración pública, con las de transporte, almacenaje, manutención y distribución a los puntos de consumo.

Resulta relevante prever y regular de antemano, es decir de modo ANTICIPATORIO, ante las circunstancias extremas de urgencia o emergencia que justifican una contratación directa (ya sea en el régimen general o con motivo de la pandemia), los parámetros relacionados con el SERVICIO DE LOGíSTICA, como integrativos de las condiciones bajo las cuales se pueden llevar adelante estas adquisiciones, el cual puede ser desarrollado por la propia Administración o contratado outsourcing, encargado del eficaz almacenamiento (centralizado o descentralizado), control de stock, distribución y entregas de los productos, en la emergencia, en los lugares que la Administración señale, a través de 
las áreas correspondientes, dentro de plazos y pautas que pueden describirse en líneas generales, con previsión de un Plan de Contingencia.

Esta debe ser una REGULACIÓN NECESARIA, más aún cuando en la presente situación de crisis sanitaria se continúan adquiriendo bienes y servicios por dicha emergencia.

Asimismo, la relevancia del tema se ve actualmente acrecentada en torno a las futuras vacunas a adquirir de los distintos laboratorios fabricantes, respecto a las cuales, recien se esta pensado en mi pais, sin proyectos concretos y con una vaga generalidad, topicos relacionados con la manutencion del stok a recibir en un futuro inmediato, con las cadenas de frio necesarias (aun no determinadas) y otros tantos aspectos que ya deberian formar parte impostergable de la agenda publica actual.

\section{CONCLUSIÓN}

Es hora entonces que la Administracion Publica, en un actuar diligente y anticipatorio regule esta tematica, como complementaria a lo establecido en el régimen general, y también complementaria a las normas especialmente dictadas de contratación directa por COVID-19.

Esa regulacion deberia involucrar aspectos esenciales generales, y cuya especificación pormenorizada sería motivo de tratamiento en los pliegos de bases y condiciones que se elaboren a los fines de integrar la documentación legal de una efectiva contratación (conforme al régimen nacional) y especificamente en los protocolos regulatorios especificos en el marco del regimen especial de COVID-19.

Ello además permitirá mayor coordinación entre las jurisdicciones contratantes y los organismos públicos y/o la sociedad destinataria de esos bienes o servicios adquiridos.

1.- RÉGIMEN ESPECIAL DICTADO PARA CONTRATAR BIENES, SERVICIOS y EQUIPAMIENTO médico, biomédico y social por COVID-19. Los Decretos de Necesidad y Urgencia del Poder Ejecutivo Nacional № $260 / 20$ (del 12/03/2020) y $N^{\circ} 287 / 20$ (del 17/03/2020) autorizan a la

Administración a no sujetarse al régimen general de contrataciones, exigiendo solamente la publicacion posterior del contrato, en la página web de la Oficina Nacional de Contrataciones.

Las Decisiones Administrativas del Jefe de Gabinete de Ministros (Nros. 409/2020 y 472/2020); las Disposiciones reglamentarias de la Oficina Nacional de Contrataciones (Nros 48/20, 53/20, 55/20) y las Comunicaciones emitidas por el mismo órgano Nros 7/20, 08/20 y 09/20, constituyen todo este marco de regulacion especial, cuyo objetivo fue obtener una mayor agilidad e inmediacion.

1.1.- Sin embargo, las falencias visibles han quedado demostradas en aspectos tales como los siguientes (a mero titulo enunciativo), a saber:

a) en torno al control de esos procedimientos;

b) en torno a los sobreprecios abonados en alimentos de primera necesidad para sectores vulnerables, en comparación con los precios de mercado;

c) en torno a la distribución de esos alimentos que no llegaban nunca a los destinatarios o lo hacían en tiempos inoportunos, circunstancia que motivó una modificación del procedimiento implementado, a través de una decisión discrecional del Ministerio de Desarrollo Social, disponiendo que los alimentos adquiridos fueran distribuidos a determinadas organizaciones intermedias (ONG, organizaciones sociales, parroquias) para que aquellas las distribuyeran a los sectores más vulnerables de la sociedad que los esperaban, pero sin indicación -sin embargo- de los instrumentos de control 
que serían aplicables, en tiempo real, a fin de verificar efectivamente la recepción de dichos bienes por sus reales destinatarios;

d) en torno a la distribución de insumos médicos y biomédicos adquiridos por el sector público para hospitales y centros de salud;

Asimismo, en los primeros meses de la pandemia no se utilizo el sistema de contratación electrónica vigente en nuestro país, hasta que -al ser comprobadas las irregularidades citadas- se comenzó a usar el sistema electrónico COMPR.AR. pero solo de modo optativo.

De igual modo, ante los sobreprecios a los que me he referido anteriormente, recien a fines de Abril del corriente año, la Jefatura de Gabinete resolvió que el Estado, en sus compras, debía someterse a los precios máximos que la Secretaria de Comercio había establecido para el comercio minorista (Decisión Administrativa № 472/2020 del 07/04/2020 y Resolución № 100/2020 Secretaría de Comercio Interior)

Finalmente, se pudo observar que los estándares de celeridad, eficacia y transparencia citados en los "Considerandos" de la normativa de contratación para COVID-19 (los cuales no solo deben abarcar la etapa de adquisición en sí, sino asimA modoismo la distribución y recepción del bien o insumo por sus destinatarios), no lograron en multiples ocasiones, concretarse en la realidad.

1.2.- Notas esenciales de este procedimiento especial de contratación directa por COVID-19.

A modo de breve sintesis, resimiremos los topicos esenciales regulados en la normativa especial:

(i) Se trata de una contratación excepcional diferente de la contratación directa por causal de emergencia del Decreto $n^{\circ}$ 1023/01. Y optativamente, los organismos pueden utilizar el sistema electrónico de adquisiciones COMP.AR hasta la realización del cuadro comparativo.

(ii) Se debe publicar en la página de la Oficina Nacional de Contrataciones el acto administrativo de conclusión del procedimiento, pues este tipo de contratación no requiere acto administrativo de autorización del llamado, ni de aprobación del Pliego de Bases y Condiciones Particulares.

(iii) Se trata de una "compulsa abreviada", mediante el envío -por correo electrónico- de tres (3) invitaciones como mínimo a tres (3) proveedores, inscriptos en SIPRO (Sistema de Informacion de Proveedores), sin plazo mínimo de antelación para cursarlas. De utilizarse optativamente el Sistema electrónico de COMPR. AR, las invitaciones se dirigen a todos aquellos inscriptos en SIPRO.

(iv) No existen requisitos de publicidad y difusión previa de la convocatoria.

(v) De no existir proveedores inscriptos en el rubro que debe adquirirse, la entidad contratante acredita esta circunstancia, y puede enviar invitaciones a otros interesados.

(vi) La Información que deben contener las invitaciones esta limitada a los siguientes datos: a) Especificaciones técnicas; b) cantidades ; c) plazo y lugar de entrega a la Administracion publica; d) día de presentación de oferta al correo institucional; e) criterio de selección; f) plazo y forma de pago; g) garantía de mantenimiento, en su caso y garantía de cumplimiento de contrato. De la LOGISTICA, nada se dice, y queda absolutamente independizada de la adquisición, sin que por otra parte, se encuentre regulada en otro sitio.

(vii) Pueden presentarse personas no invitadas. 
(viii) Si se presume que los oferentes concertaron posturas o media simulación de competencia, o incumplimientos en contratos previos, las ofertas serán rechazadas o el contrato se rescindirá de pleno derecho.

(ix) La Sindicatura General de la Nacion (organo de control interno del Poder Ejecutivo Nacional) solo interviene si el monto estimado supera un determinado límite.

(x) No hay etapa de evaluación de ofertas en sentido formal:

-No interviene una Comisión Evaluadora.

-No se emite Dictamen de Evaluación.

-El titular de la Unidad Operativa de Contrataciones emite un Informe recomendando la resolución a adoptar.

(xi) Si el procedimiento resulta fracasado o desierto, se puede realizar una selección en forma directa. En caso de rescisión por culpa del proveedor, no se envían los antecedentes a la Oficina Nacional de Contrataciones, pues no resulta aplicable el régimen sancionatorio del Reglamento General de Contrataciones del sector público nacional.

1.3.- De la síntesis realizada, devienen varias reflexiones:

I.- La falta de publicidad de las convocatorias impide una participación mayor de oferentes.

II.- Al no existir etapa de evaluación, atento a que el propio titular del ente contratante es el que recomienda adjudicar, se conspira contra la transparencia, circunstancia que tambien ocurre al ser meramente optativa la utilizacion del sistema electrónico de contrataciones de bienes y servicios.

III.- No existe control por parte de los organismos de control en tiempo real. El acto de conclusión del procedimiento se publica en la página web de O.N.C., pero no hay sitio en el cual se reúnan todas las compras en formatos abiertos.

IV.- Teniendo en cuenta que cada organismo puede decidir usar este sistema especial de contratación, existe un margen muy abierto de discrecionalidad para su utilizacion, aún en situaciones que no ameritan quizás su empleo (no hay listado de bienes o servicios que se pueden adquirir por ese sistema).

V.- Las sanciones que se apliquen a los proveedores, aún la rescisión del contrato, los coloca en situación de privilegio respecto del resto de los co-contratantes del Estado, regidos por el Régimen general de contrataciones del Estado Nacional, puesto que expresamente se prevé no remitir los antecedentes a la ONC, por la mera circunstancia de tratarse de contratación por emergencia por COVID-19.

Por ultimo, ademas cabe concluir que los servicios de logística no han integrado este marco especial regulatorio, OMISIÓN que es RELEVANTE ante las realidades vividas frente a las necesidades a cubrir.

2.- Recomendaciones de Transparencia Internacional en torno a contrataciones publica en situaciones de emergencia ${ }^{2}$.

https://www.transparency.org/es/press/coronavirus-sparks-high-risk-of-corruption-across-latinamerica. Transparencia Internacional identifica riesgos de corrupción y medidas de integridad en el marco de COVID-19 (26/03/2020). 
Para concluir, resulta interesante poner un foco en las recomendaciones emititas por "Transparencia Internacional", relacionadas con las contrataciones públicas en situaciones de emergencia, que estimo relevantes para ser tenidas en cuenta frente a algunas debilidades emergentes del sistema especial de contratación por COVID-19 implementado a nivel nacional.

Las recomendaciones aludidas en periodos de emergencia, se centran, fundamentalmente, en cinco tópicos que las Administraciones Publicas deben observar:

I.- En primer lugar, la importancia de brindar una "información continua, veraz, verificable y en lenguaje ciudadano" sobre las compras y contrataciones, en plataforma de datos abiertos, accesibles y concentradas en sitio público específico, que incluya informes sobre las auditorías realizadas.

II.- En segundo lugar, la necesidad de "administrar el presupuesto para atender las emergencias por casos de fuerza mayor, bajo los principios de eficiencia, eficacia, economía, transparencia e imparcialidad". Ello exige auditorías en tiempo real, para asegurar el cumplimiento de los principios básicos que rigen las contrataciones públicas.

III.- En tercer lugar, la necesidad de "rendiciones de cuentas, al término de la emergencia, con participación ciudadana y existencia de mecanismos independientes de monitoreo de esos contratos".

Aquellas rendiciones de cuentas también deben visibilizar la

relacion entre necesidades a cubrir y el estatus de cumplimiento de cada una de ellas.

IV.- En cuarto lugar, la necesidad de evitar la concentración en los procedimientos de contratación, y para ello fomentar la participación de las PYMES, utilizando convenios marco si los hubiera, indicando precios máximos, a fin de prevenir las prácticas desleales.

V.- En quinto lugar, la necesidad de implementar mecanismos adicionales de control a los previstos en las normativas nacional y locales, con protocolos de actuación de los órganos de fiscalización respecto al destino de los recursos, en tiempo real, asi como al análisis del cumplimiento de los objetivos perseguidos conforme a las necesidades a satisfacer.

En forma simultánea, tambien se recomienda la neesidad de implementar canales de denuncia ciudadana.

Estas recomendaciones resultan apropiadas, razonables y valiosas y deben ser consideradas por la Administración Pública nacional y Administraciones provinciales, a fin de mejorar el régimen especial de contratación por emergencia de COVID-19, superando los aspectos endebles que aquel sistema ha demostrado.

Al mismo tiempo, resultan un marco relevante para introducir un aspecto olvidado: la regulación de la logística, que necesariamente y en un actuar PROACTIVO y ANTICIPATORIO respecto a las futuras contrataciones por la pandemia, debera complementar la normativa especial dictada para adquirir bienes y servicios imprescindibles para afrontar la crisis sanitaria, y satisfacer, en tiempo oportuno y con eficacia, las necesidades y objetivos involucrados en aquellas. 